\title{
Calembours poétiques et traduction : Glossaire j'y serre mes gloses, de Michel Leiris à André Masson
}

Fabio Vasarri

\section{(2) OpenEdition}

\section{Journals}

Édition électronique

URL : http://journals.openedition.org/rief/1305

DOI : 10.4000/rief.1305

ISSN : 2240-7456

Éditeur

Seminario di filologia francese

\section{Référence électronique}

Fabio Vasarri, «Calembours poétiques et traduction : Glossaire j'y serre mes gloses, de Michel Leiris à André Masson », Revue italienne d'études françaises [En ligne], 6 | 2016, mis en ligne le 15 décembre 2016, consulté le 19 avril 2019. URL : http://journals.openedition.org/rief/1305 ; DOI : 10.4000/ rief. 1305

Ce document a été généré automatiquement le 19 avril 2019.

\section{c) (i) $९$}

Les contenus de la RIEF sont mis à disposition selon les termes de la Licence Creative Commons Attribution - Pas d'Utilisation Commerciale - Pas de Modification 4.0 International. 


\title{
Calembours poétiques et traduction : Glossaire j'y serre mes gloses, de Michel Leiris à André Masson
}

\author{
Fabio Vasarri
}

\section{NOTE DE L'ÉDITEUR}

En ce qui concerne la reproduction des images, l'éditeur a essayé de se mettre en contact avec les ayants droit sans toutefois y parvenir. L'éditeur reste donc à la disposition.

1 La notion d'intraduisibilité est suspecte et demande bien des précautions, mais on aurait du mal à contester que Glossaire j'y serre mes gloses (1939) de Michel Leiris est un recueil de jeux de mots foncièrement intraduisible dans n'importe quelle langue.

Dans L' Âge d'homme, Leiris a défini ces jeux de mots en forme d'articles de dictionnaire comme des calembours poétiques ${ }^{1}$. Il y exploite en effet le vocabulaire en tant que réservoir d'images lyriques et de révélations surprenantes. L'enjeu de cette activité à la fois ludique et thaumaturgique, inspirée notamment par l'exemple de Robert Desnos et poursuivie tout au long de la vie de l'auteur, est d'ordre poétique (réactiver le langage atrophié par l'usage courant) mais aussi existentiel, puisqu'il s'agit rien moins que de « [1]aisser les mots s'animer, se dénuder et nous montrer par chance [...] quelques-unes de nos raisons de vivre et de mourir $»^{2}$. Et ce sera encore à partir de sa pratique singulière du calembour que Leiris édifiera son testament poétique, l'étonnant « Musique en texte et musique antitexte» de $1985^{3}$, où cette pratique est à la fois l'objet d'un art poétique détaillé et le sujet d'un tour de force stylistique, puisque l'écriture de ce mémoire est à son tour émaillée de paronomases et d'homophonies.

Dans le pseudo-dictionnaire, la définition du mot-entrée ne doit rien à l'étymologie et aux acceptions admises, elle crée un nouveau sens à partir de la matière phonique et 
graphique du vocable. Voici quelques exemples avec les dates de publication et les procédés correspondants :

révolution - solution de tout rêve ? (« analyse ») $)^{4}$ [1925]

vie - un Dé la sépare du viDe (syncope) [1926]

désir - désert irisé (" analyse ») [1939]

désir - « rides » inversé ? (anagramme) [1985]

Dans ce lexique poétique, les mots-entrées sont donc suivis de ce que l'auteur appelle gloses, c'est-à-dire de définitions sous forme de calembours essentiellement phoniques. Les premières listes de vocables ont paru dans La Révolution surréaliste de 1925 à 1926 . Or la poétique surréaliste tend à exclure l'idée même de traduction mentale de la pensée dans le langage ; comme le dit Tristan Tzara, la pensée se fait dans la bouche et non pas dans la tête. Le mirage du texte automatique qui court-circuiterait le logos pour atteindre les profondeurs psychiques exclut cette notion de traduction.

5 Pour ce qui est du calembour poétique, Leiris est toujours resté fidèle au précepte de Tzara ; en 1985, il le cite encore et il définit son glossaire comme une machine à penser ${ }^{5}$. D'ailleurs, il n'est pas un auteur particulièrement attiré par les problèmes de la traduction interlinguistique, comme tant d'autres; du moins, il n'a pas laissé de réflexions articulées à ce sujet ${ }^{6}$. Pourtant, un mouvement traductif traverse Glossaire j’y serre mes gloses dans plusieurs directions, qui se trouvent correspondre au classement ternaire de Jakobson ${ }^{7}$. Cela tient d'abord au projet même du recueil, qui prétend donner la définition juste, cratylienne du vocable, en corrigeant l'arbitraire du sens commun que propose le dictionnaire. Tout le glossaire constituerait donc, à la rigueur, une opération de traduction poétique intralinguistique, du français au français. Mais on y trouve aussi des cas plus classiques de traduction interlinguistique, selon la formule "entrée étrangère - glose française ». La troisième dimension traductive que je retiendrai est intersémiotique, et elle concerne moins les calligrammes de Leiris que les lithographies d'André Masson qui ornent l'édition originale de $1939^{\circ}$. Les deux séries alternées des calligrammes et des lithographies constituent un dispositif visuel double dans cette plaquette. Elles contrebalancent en quelque sorte le côté intraduisible du glossaire, en proposant une version iconique. C'est sur les lithographies de Masson que je m'attarderai, non seulement parce que ces dessins, souvent remarquables, sont peu connus et peu étudiés, mais aussi parce que, contrairement aux autres cas mentionnés, ils supposent une confrontation réelle de deux sujets, le poète et le peintre, et le problème de la traduction visuelle du jeu de mots.

\section{Traduction intralinguistique}

6 De toute évidence, le sens admis du mot "glossaire » implique la traduction : recueil d'acceptions particulières à l'intérieur d'une langue ou d'un jargon, mais aussi recueil de mots exotiques ou anciens. Il est vrai que Leiris subvertit le modèle, puisque chez lui l'exotisme est partout, dans l'entrée et aussi dans la glose. Toujours est-il que celle-ci se présente comme la traduction intralinguistique d'un mot isolé; en particulier, dans les exemples monoverbaux, la glose mime l'article de dictionnaire des synonymes, un dictionnaire où les paronymes prétendraient supplanter les synonymes.

7 Pour qu'il y ait effet traductif, il convient que l'entrée et la glose appartiennent à la même catégorie grammaticale, par exemple deux substantifs : 
avenir = navire.

penser, serpenter.

rixe, risque.

sceptre $=$ spectre $^{9}$

Ici, la glose pose une équivalence, comme dans une traduction. Et même dans les cas mixtes, pour l'essentiel "substantif = adjectif», elle peut isoler un trait sémantique capital qui aspire à une valeur générale (exemple : « secret sacré », de 1985). Quoi qu'il en soit, le statut traductif est ici, on le voit, approximatif et tout extérieur. Quant à la question de la traduction « interne », mentale et pas seulement intralinguistique, ce n'est pas mon propos $\mathrm{ici}^{10}$.

9 Mais il importe de signaler ce mouvement à l'intérieur d'une seule langue, le français, mouvement qui propose à chaque fois le sens profond, anti-conventionnel du mot. On pourrait aussi retenir, dans la perspective intralinguistique, les gloses par épellation lexicalisée (selon la formule de Gérard Genette) ${ }^{11}$, qui traduisent la chaîne phonétique des lettres dans des séquences inouies et oraculaires :

homme - à chaud, aime et meut.

néant - est né à haine, hanté.

Dans quelle langue ces formules sont-elles écrites? Sans doute, dans cette « langue de la mort ", lapidaire et dépaysante, dont il est question dans " Musique en texte ». André Masson a aussi relevé le côté métaphysique de Glossaire j'y serre mes gloses ${ }^{12}$. En exploitant l'épellation, qui se fonde sur une véritable contrainte formelle, Leiris en arrive à forcer l'usage et les règles syntaxiques du français et à créer en quelque sorte une autre langue. Cela par fidélité à un credo concernant le «vrai » langage et son noyau poétique. On songe presque à la notion benjaminienne de la "pure langue » idéale que toute traduction devrait laisser transparaître ${ }^{13}$. Ce qui semble évident, c'est que ce pseudodictionnaire exhibe au grand jour la démarche même du texte lyrique : le passage de la langue à la parole, du code au message, du sociolecte à l'idiolecte.

\section{Traduction interlinguistique}

11 Mais Leiris s'aventure aussi dans Babel, en proposant des équivalents poétiques français de mots étrangers, surtout allemands et anglais. Il a dû hésiter à élargir de la sorte le champ du glossaire, puisque ces gloses bilingues apparaissent à partir de la plaquette de 1939, où elles sont assez nombreuses (une vingtaine), et qu'elles s'espacent par la suite. L'hypotexte des pseudo-définitions est dans ce cas le dictionnaire bilingue. Cela ressort de nouveau dans les exemples monoverbaux, qui miment la concision du dictionnaire de poche et la monosémie :

dream $=$ drame.

L'activité onirique, dont on connaît le rôle central dans la lignée romantique-surréaliste, en vient à constituer une sorte de théâtre mental. Ailleurs, Leiris décompose le mot étranger :

nevermore - ... aux névés morts ( = aux calendes grecques).

Ici, le mot-thème est chargé de réminiscences littéraires (le refrain du Corbeau de Poe, repris dans les titres de deux poèmes saturniens de Verlaine). L'exactitude mathématique de la formule (nevermore = névés morts) produit un syntagme dépaysant mais qui respecte 
le sens de l'adverbe anglais: quand les neiges éternelles fondront, c'est-à-dire jamais ( «jamais plus » dans les traductions de Baudelaire et de Mallarmé). Or, Poe lui-même l'a noté et Eco l'a rappelé, c'est surtout le «o » long suivi de « $\mathrm{r}$ » qui donne à nevermore sa sonorité prolongée et lugubre ${ }^{14}$. Contrairement aux versions des deux poètes, le calembour de Leiris garde ce trait essentiel.

Un supplément d'explication est pourtant nécessaire ( $=$ aux calendes grecques »). Tout se passe en effet comme si la traduction interlinguistique partait d'une équivalence phonique qui a besoin d'être complétée par un ajout d'ordre purement sémantique, sans rapport avec la matière sonore de l'entrée :

die Wolken - les volcans d'eau.

zufrieren - souffrir de neige.

Dans la deuxième glose, le mot «neige » n'est que faiblement justifié selon le principe paronymique du recueil; il complète la traduction interlinguistique en assurant la communication du message. L'accent se déplace en quelque sorte de la langue-source à la langue-cible, tout en gardant la métaphore première, à savoir que geler (zufrieren), c'est souffrir, ou que les nuages (die Wolken) sont des volcans renversés déversant la pluie.

Un mot étranger isolé peut aussi à l'occasion s'insinuer dans la langue de la mort, comme dans la version la plus récente (1986) de la glose du mot «fantôme » («fichu hante-home !»). Il n'y a pas jusqu'à la rétro-traduction qui manque à l'appel. Il s'agira alors d'une entrée française et d'une glose étrangère, que ce soit sur le mode provocant de l'antinationalisme ("drapeau - der Popo », le derrière) ou sur celui d'un commentaire plus personnel :

elle - hell, « enfer » ou " clair », - en anglais, ou en allemand.

Cette entrée fait partie de la série des pronoms personnels, bien représentés dans Glossaire. Exemple non pas bilingue mais trilingue, qui retient simultanément des signifiés distants voire opposés pour exprimer toute l'ambivalence du rapport à la femme.

Il faudrait considérer aussi les gloses de noms propres étrangers (Shakespeare, Verdi, Wagner etc.), qui abondent dans le recueil de 1985 : le jeu de Leiris ne s'arrête pas devant ce qu'on ne traduit pas normalement, les patronymes ou les toponymes... Dans l'ensemble, ces calembours interlinguistiques montrent que Babel et Cratylie ne s'opposent pas forcément, qu'elles peuvent même être solidaires. Ce qui implique que toutes les langues ont un fond commun, alors que leur pluralité est depuis Hermogène, l'anti-Cratyle du dialogue de Platon, une preuve de l'arbitraire du langage ${ }^{15}$. Sur le plan qui nous intéresse de plus près, ces gloses montrent une solution certes utopique des impasses de la traduction poétique : en gardant la sonorité du mot étranger, on pourra tout de même transporter quelque chose de son sens, voire découvrir son sens profond. À propos des étymologies populaires de mots anglais, commentées par Mallarmé, Genette parle de «subversion ironique [...] du concept même de traduction $»^{16}$. Il y a un peu de cela chez Leiris, mais aussi et surtout une démarche utopique et constructive de remotivation poétique.

\section{Traduction intersémiotique}

19 Les dix calligrammes que Leiris a insérés dans la plaquette de 1939 témoignent d'une dimension intersémiotique. Mais ces poèmes-figures échappent à la règle lexicographique du glossaire et on a du mal à reconnaître le mot isolé dont les autres mots constitueraient 
la glose ; on a également du mal à repérer un hypotexte, en l'occurrence une phrase dont le calligramme serait la traduction visuelle. Leur dimension traductive se réduit à la transposition graphique du jeu de mots, puisque la matière phonique est forcément absente. On reste dans un entre-deux langage-image, sans transformation finale. Le dispositif iconique est formé par le calligramme et par le titre, qui est plutôt une légende. L'entrelacement des mots dessine des formes qui découlent moins d'une préméditation que d'une construction progressive, voire d'une heureuse coïncidence : « La fronde », « La hache ", «Le sceptre miroitant »... En tout cas, contrairement à ce qui arrive dans le dessin ou dans la peinture, les deux composantes du dispositif sont homogènes, linguistiques et plus précisément graphiques ${ }^{17}$.

Quant à la relation intersémiotique à proprement parler, c'est Leiris lui-même qui la suggère, sous forme d'un parallèle entre la poésie et la musique. Dans «Musique en texte ", en essayant de définir l'idiome de ses gloses, il a recours à deux comparants sonores, les vocalises d'opéra e le scat singing du jazz vocal, qui illustrent la désarticulation du langage. Mais c'est bien sur le plan iconique, grâce à la collaboration avec Masson, que cette relation se met en place ${ }^{18}$.

On ne sait pas grand-chose sur cette collaboration pour la plaquette de 1939. Les deux amis étaient liés depuis 1922, et Leiris fréquentait les ateliers avoisinants d'André Masson et de Joan Miró rue Blomet, à Paris. Tant Leiris que Masson ont écrit des témoignages sur cette époque effervescente et sur le groupe d'amis (entre autres, Antonin Artaud et Georges Limbour) qui se réunissait autour des deux ateliers. Il convient de rappeler pour notre propos que ce groupe, bien que proche du surréalisme, s'en distinguait par ce que Leiris appelle son « souci compositionnel »; autrement dit, les productions artistiques des amis de la rue Blomet faisaient preuve de préoccupations formelles, de règles voire de contraintes, s'opposant par-là à l'automatisme ${ }^{19}$. Par ses mots et par ses images, Glossaire j'y serre mes gloses peut bien illustrer ce trait spécifique.

22 La publication de la plaquette a été retardée d'une dizaine d'années à cause des problèmes financiers de l'éditeur, Daniel-Henri Kahnweiler, et sans doute aussi à cause d'une brouille entre celui-ci et Masson ${ }^{20}$. Quant au choix des entrées à illustrer, on n'en sait guère plus ; on constate que les quinze lithographies se disposent assez régulièrement selon la distribution alphabétique ${ }^{21}$.

Françoise Will-Levaillant, spécialiste du peintre, relate que l'écrivain n'était pas toujours d'accord sur les titres des lithographies, ce qui pourrait être un indice intéressant face à des illustrations parfois très libres vis-à-vis du texte, mais il paraît que le désaccord ne concernait que l'aspect typographique de ces titres ${ }^{22}$.

24 Masson avait illustré le recueil Simulacre (1925) pour le même éditeur. Miró illustrera Bagatelles végétales (1956), autre recueil de jeux de mots de Leiris ; mais, dans les deux cas, les illustrations ne portent pas de titres et sont tout à fait libres vis-à-vis des textes. Il en va autrement pour le glossaire, qui pose la question du rapport à l'écrit et donc de la traduction visuelle : à part la première, qui orne la couverture, chaque lithographie est pourvue d'un intitulé qui correspond à un mot-entrée.

Cela ne veut pas dire que Masson adhère toujours au texte; de fait, il n'assume qu'en partie la contrainte de transposer sur le plan iconique les jeux linguistiques. Il y a une tension manifeste entre cette contrainte et la création autonome. On distinguera, d'une manière assez classique ${ }^{23}$, trois démarches de l'illustrateur : la littéralité, axée sur le mot- 
entrée, la fidélité au texte de la glose, axée donc sur celui-ci et la recréation libre, produisant de nouveaux jeux visuels.

\subsection{Littéralité}

Certaines lithographies se rapprochent de l'illustration traditionnelle, en présentant une image figurative du mot-entrée ; c'est le cas d' « Assassinat ", «Lune », " Métamorphose » ou « Ruine ». Le rapport avec la glose reste très vague. Par exemple, à l'article « lune » («l'urne des nues» selon Leiris), correspond une sorte de polyptyque à quatre compartiments représentant la lune associée sans doute aux quatre éléments fondamentaux, air, terre, eau et feu. Quant à la notion, chère aux surréalistes, de "métamorphose ", elle donne lieu dans le dessin correspondant à une transmutation visuelle de formes humaines et animales, alors que Leiris construit des formules oraculaires ${ }^{24}$. Ces images peuvent être saisissantes, mais elles éludent ou ratent le rapport avec l'hypotexte.

Ce qu'en revanche il importe de souligner, c'est la subdivision à la Magritte de l'image, que l'on trouve dans «Lune", mais aussi dans «Assassinat» et dans «Femme ». Cet expédient peut renvoyer en effet à un principe fondamental du glossaire, la décomposition des mots. Dans le dernier cas mentionné, Leiris propose une équation mathématique ( femme - flamme sans aile?», soit «femme = flamme - L»), que l'on comprend mieux si l'on tient compte que, dans son imaginaire alphabétique, «l» est une consonne masculine ${ }^{25}$. Masson, de son côté, découpe le corps féminin en quatre de haut en bas et le dédouble de gauche à droite [Figure 1]. Il en résulte un blason bipartite et spéculaire, où l'on remarque la distinction humain/végétal. La relation avec la matière verbale reste vague, mais la dissection anatomique fait écho à celle que le poète pratique sur le langage.

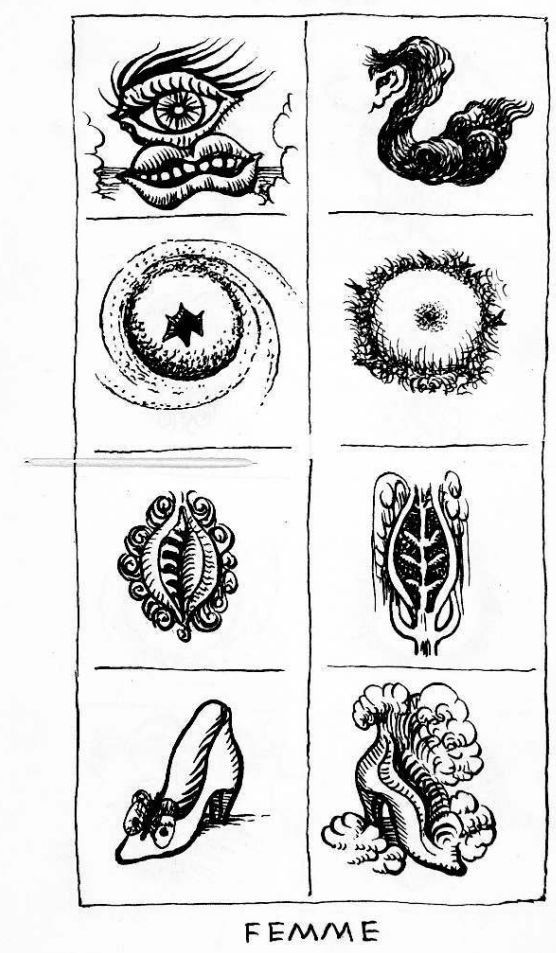

Fig. 1. André Masson, « Femme», lithographie, dans Michel Leiris, Glossaire j'y serre mes gloses (1939). 


\subsection{Fidélité} cette lithographie [Figure 2] éclaire et parachève le sens allusif de la glose : celle-ci évoque le « sommeil de midi, demi-nid, demi-hyène... ", bref une notion très ambiguë que l'on peut rattacher au démon de midi et au fantasme érotique. Masson concrétise pour ainsi dire ceux-ci dans un être indécidable et troublant qu'il montre à la fois de face et de dos.

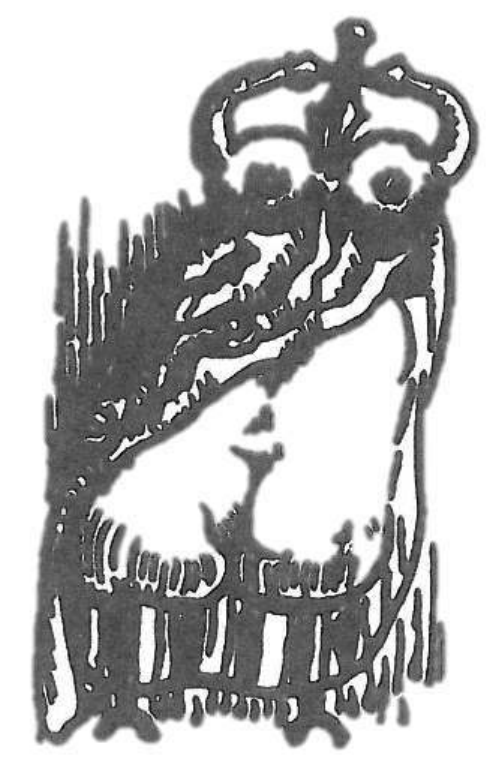

Fig. 2. André Masson, « Méridienne », lithographie, dans Michel Leiris, Glossaire j'y serre mes gloses (1939).

\subsection{Recréation}

Pour le mot "girasol», Leiris associe la fleur à une girafe, tandis que Masson trace un phallus végétal, qui, comme le cou de cet animal, «s'érige haut ». Mais il y a surtout deux lithographies où l'artiste exploite des calembours qui manquent dans les gloses correspondantes, en restituant en quelque sorte sur le mode du rébus des procédés très fréquents dans les jeux de mots (polysémie, homophonie). Il s'agit des dessins intitulés «Pensée » et « Signe », dont l'importance à l'intérieur du recueil peut être confirmée par le fait que ce sont les seuls qui ont fait l'objet d'une prépublication ${ }^{26}$. Dans ces cas, les illustrations de Masson rappellent donc le modèle du rébus, tout comme les gloses de 
Leiris peuvent évoquer les définitions de mots croisés. Sans exclure la subversion et l'humour, c'est le côté énigmatique du glossaire qui ressort de la sorte, tant dans les mots que dans les images.

Dans la glose correspondante de 1939, la pensée « s'épand sans cesse, ou bien s'empèse ». Leiris a donné d'autres définitions à l'intérieur de ce champ lexical. En particulier, il existe deux gloses de l'infinitif penser: on a cité la première, de 1939, monoverbale ("serpenter »); celle de 1985, plus pessimiste ("s'empeser, s'empêtrer?»), reprend le deuxième paronyme de "pensée ", c'est-à-dire "s'empeser ». Bref, dans l'ensemble, plusieurs signifiés se croisent : l'épanouissement ou au contraire la menace de la rigidité et de l'impasse ; plusieurs référents aussi : le serpent et, d'une manière plus indirecte, la fleur (puisque la pensée «s'épand » comme une fleur qui s'épanouit).

Ce dernier aspect devient explicite et central chez Masson [Fig. 3]. Son image se base sur la polysémie du substantif "pensée ", activité mentale et fleur, ainsi nommée car elle suscite des réflexions et évoque des souvenirs. Sur le plan visuel, c'est une bouche contenant une fleur. Bouches et fleurs reviennent, séparées cette fois, dans la lithographie qui orne le frontispice de la plaquette et, à nouveau réunies, dans une autre œuvre de Masson, le mannequin "La jeune fille au ballon vert à bouche de pensée", présenté à l'Exposition Internationale Surréaliste de Paris en $1938^{27}$. Il s'agit donc d'un motif récurrent, dont les échos littéraires sont forts, de la lettre dite du voyant de Rimbaud ("j'assiste à l'éclosion de ma pensée ») à l'adage de Tzara mentionné plus haut, «la pensée se fait dans la bouche ».

Ce dessin a son pendant dans l'illustration de l'entrée lèvres qui mêle à son tour la bouche et la fleur [Fig. 4]. Plus précisément, le ver que l'on voit en haut suggère le caractère végétal du bord de l'image, et cette bouche-fleur (ou bouche-fruit?) contient d'autres bouches, conformément au pluriel qui domine la glose («lèvres, on les lit comme des livres»). On songe aussi à la métonymie que l'on trouve, comme le rappelle Jacques Derrida, dans le passage de la Genèse sur Babel: en hébreu, on ne dit pas "langue » pour « idiome ", mais bien " lèvre ", au singulier ${ }^{28}$.

À part la valeur érotique de ces lèvres, plausible puisque l'éros imprègne l'œuvre de Masson, on peut donc supposer que le peintre a voulu étendre le jeu en présentant deux images analogues. La seconde ("Lèvres») pourrait contenir une allusion à Babel, aux lèvres, donc aux langues multiples emboîtées les unes dans les autres, puisqu'à l'origine le langage était un et universel. De son côté, Leiris rattache les livres aux lèvres, l'écrit à la phoné. C'est toujours dans la bouche - et dans l'oreille - que cela se passe. 

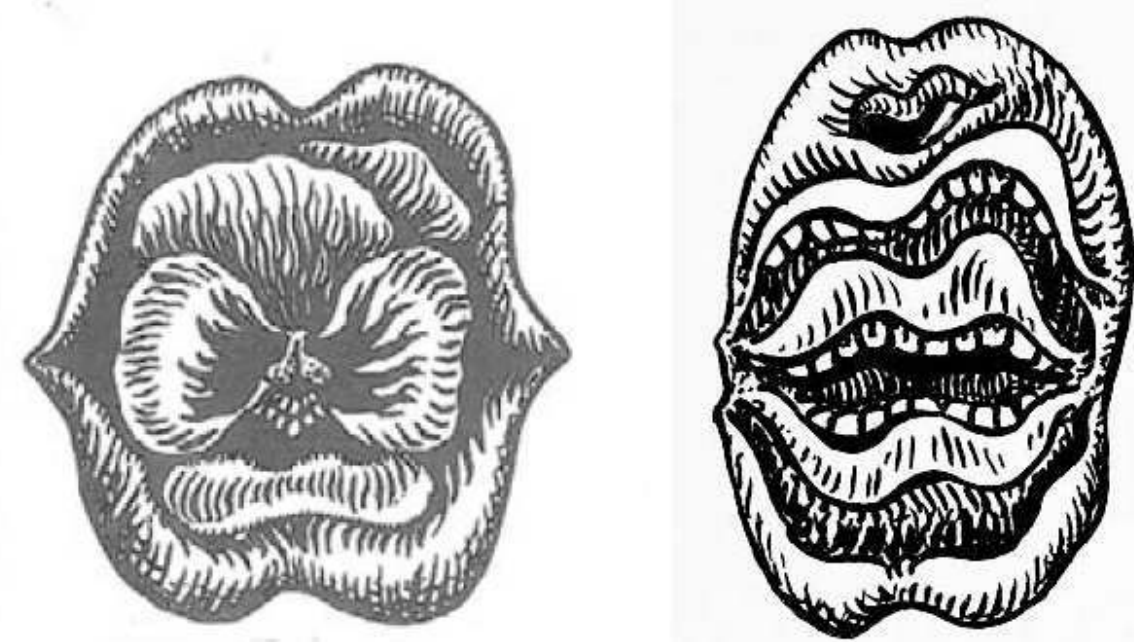

Fig. 3 (à gauche). André Masson, «Pensée », lithographie, dans Michel Leiris, Glossaire j'y serre mes gloses (1939)

Fig. 4 (à droite). André Masson, « Lèvres », lithographie, dans Michel Leiris, Glossaire j'y serre mes gloses (1939).

Dans «Signe» [Fig. 5], la liberté et la recréation s'accentuent encore. Leiris pose le rapport cratylien entre le signe et le référent, dans une glose qui est devenue assez célèbre grâce au commentaire de Genette : « signe - il singe ». Le mot imite la chose, il est donc motivé. Le dessin de Masson, lui, ignore cette métathèse au profit d'un homophone, lui aussi animal. Autrement dit, il y a un double glissement, du signe au singe et au cygne.

Il va sans dire que l'oiseau blanc est chargé ici de réminiscences littéraires, notamment de Baudelaire et de Mallarmé. Mais Masson personnalise la métaphore usée du cygne-poète. Le lac de Mallarmé devient une sorte de marécage. L'oiseau métamorphique, dont le corps se termine en queue de poisson, se trouve hors de l'eau comme dans le poème de Baudelaire. L'œil qui campe au milieu est fréquent chez l'artiste, et on le retrouve dans la lithographie "Caillou» de la plaquette. Qui plus est, quelques textes de Masson contemporains du dessin, qui sont de fait pour l'essentiel des didascalies d'autres dessins, présentent des images analogues: "rives de l'ennui», «signes de feu», « œil du marécage » et aussi, «le soleil, comme un œil pensant ${ }^{29}$. Donc, on pense aussi par le regard. 


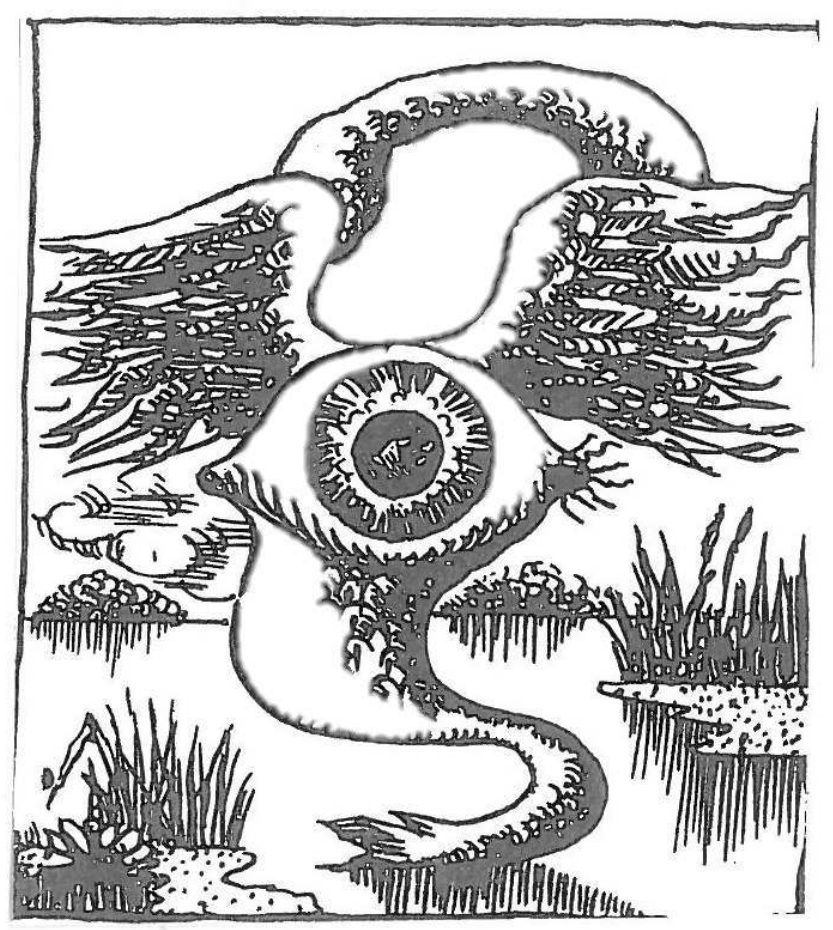

Fig. 5. André Masson, « Signe », lithographie, dans Michel Leiris, Glossairej'y serre mes gloses (1939).

Ce cygne marque le point où Masson s'écarte le plus de Leiris, en respectant la règle du jeu de mots mais en proposant une image alternative. Ce n'est pas un hasard si Lawrence Saphire, spécialiste de l'artiste, a vu dans son travail d'illustrateur une œuvre relativement autonome, "son propre glossaire» de sa seconde période surréaliste $(1937-1943)^{30}$. Le paradoxe c'est que, sur un plan général, c'est l'image figurative qui singe l'objet représenté et qui lui ressemble, tandis que le signe linguistique doit s'accommoder d'un cratylisme secondaire. On dirait que les rôles s'inversent dans l'opposition d'un poète mimétique et d'un peintre voyant.

Leiris vise à corriger l'arbitraire du signe linguistique et à le motiver. L'image, elle, a un rapport de ressemblance avec l'objet représenté et elle est donc traditionnellement motivée. Mais le portrait n'est pas le référent et le signe iconique a aussi ses conventions, tout comme le langage : c'est, entre autre, l'enseignement de Magritte ${ }^{31}$. De fait, Masson rompt ces conventions et dessine autre chose.

9 Tout compte fait, les démarches du poète et de l'illustrateur du Glossaire sont solidaires : rupture radicale des conventions et création d'un espace métaphorique et métaphysique. Quoi qu'il en soit, bien plus tard, Leiris reprendra dans "Musique en texte " les deux gloses sans le mot-entrée "signe », en se définissant comme "cygne et singe », poète et pitre, ce qui est une manière de boucler la boucle ${ }^{32}$.

Le "peintre-poète " ${ }^{33}$ Masson travaille pour, avec et parfois même contre son ami écrivain, en assumant jusqu'à un certain point le défi de transposer en images les jeux de la matière verbale. Mais les tensions multiples qui se croisent dans la plaquette de 1939 accroissent sa valeur et aussi sa saveur : les tensions entre le poète et l'artiste, le mot et l'image, la liberté et la contrainte, le surréalisme d'aujourd'hui et l'Oulipo de demain. Loin d'en être exclue comme il pourrait sembler à première vue, la traduction, métamorphose et transmission, est bien installée dans ce champ de forces. 


\section{NOTES}

1. M. Leiris, L'Âge d'homme, Paris, Gallimard, "Folio », 1973, p. 193. Je rappelle que, après les prépublications partielles dans La Révolution surréaliste (1925-1926), deux recueils principaux des "gloses » leirisiennes ont vu le jour: Glossaire j’y serre mes gloses, illustré par A. Masson, Paris, Éditions de la Galerie Simon, 1939, repris sans les illustrations dans Mots sans mémoire, Paris, Gallimard, 1969, p. 71-116 et "Souple mantique ou simples tics de glotte en supplément », dans Langage tangage ou ce que les mots me disent, Paris, Gallimard, 1985, p. 7-68.

2. M. Leiris, prière d'insérer de Glossaire j'y serre mes gloses (1939), dans Brisées, Paris, Mercure de France, 1966, p. 69. Sur les péritextes du recueil voir G. Poitry, «Le Glossaire de Michel Leiris ou "la Poésie joue son jeu" ", dans Désordre du jeu-poétiques ludiques, J. Berchtold, C. Lucken et S. Schoettke (dir.), Genève, Droz, 1994, p.153-160. Les études sur le Glossaire sont désormais nombreuses, comme le prouvent les sections bibliographiques de P.-H. Kléber, Glossaire j'y serre mes gloses de Michel Leiris et la question du langage, Paris, L'Harmattan, 1999 et de M. Leiris, Glossaire j'y serre mes gloses, illustré par A. Masson, suivi de Bagatelles végétales, illustré par J. Miró, éd. de L. Yvert, Paris, Gallimard, « Poésie », 2014. On rappellera ici en particulier M. Calle-Gruber, «Anamorphoses textuelles», dans Poétique, 42, avril 1980, p. 234-249; M.-P. Berranger, Dépaysement de l'aphorisme, Paris, Corti, 1988, p. 174-203 et, pour le contexte, J. de Sermet, Michel Leiris, poète surréaliste, Paris, P.U.F., 1997.

3. M. Leiris, "Musique en texte et musique antitexte", dans Langage tangage ou ce que les mots me disent, cit., p. 69-189. J'ai abordé autrefois cette poétique du jeu de mots leirisien, voir « Leiris : la prova del suono ", dans Saggi e ricerche di letteratura francese, XXVII, 1988, p. 213-239.

4. Ou analyse syntagmatique. Je reprends la formule saussurienne utilisée par G. Genette, Mimologiques, Paris, Seuil, 1976, p. 18-20 et 368.

5. Voir M. Leiris, Langage tangage ou ce que les mots me disent, cit., p. 105, 110 et 125.

6. Voir pourtant les remarques sur l'altérité linguistique, Ibid., p. 80-81.

7. R. Jakobson, « Aspects linguistiques de la traduction », dans Essais de linguistique générale [1959], Paris, les Éditions de Minuit, 1963, p. 78-86.

8. Elles sont à nouveau disponibles dans M. Leiris, Glossaire j'y serre mes gloses, éd. de L. Yvert, cit. Cette édition réunit en ordre alphabétique toutes les gloses de Leiris; dans mes citations, je supprimerai donc les renvois aux pages. La reproduction des illustrations, que j'ai confrontée avec l'édition originale de 1939, est fiable. La mise en page est bien sûr différente, à cause du nombre plus important des entrées. Pour des précisions intéressantes sur la genèse de l'ouvrage voir aussi C. Maubon - L. Yvert, «Le manuscrit Doucet du Glossaire de Michel Leiris ", Doucet Littérature, page publiée le 19 avril 2012, mise à jour le $1^{\mathrm{er}}$ mars 2014, consultée le 26/10/2016, URL : <http://www.doucet-litterature.org/spip.php?article61>.

9. Sauf indication contraire, toutes les gloses citées dorénavant proviennent de l'édition originale de 1939.

10. Voir entre autres G. Steiner, Après Babel [1975], Paris, Albin Michel, 1998, p. 29-90 et P. Ricœur, Sur la traduction, Paris, Bayard, 2003.

11. G. Genette, « Signe : singe », dans Mimologiques, cit., p. 351-375 (en particulier p. 368).

12. Voir la lettre de Masson du 24 mai 1939 à Daniel-Henri Kahnweiler, citée dans M. Leiris, Glossaire j'y serre mes gloses, éd. Yvert, cit., p. 31 et cf. Langage tangage ou ce que les mots me disent, cit., en particulier p. 108-110.

13. W. Benjamin, « La tâche du traducteur » [1923], dans CEuvres, I, Paris, Gallimard, 2000. 
14. Voir E. A. Poe, The Philosophy of Composition [1846], cité et commenté par U. Eco, Dire quasi la stessa cosa, Milano, Bompiani, 2003, p. 285-296.

15. Voir G. Genette, op. cit., en particulier p. 268-272.

16. Ibid., p. 261.

17. Je reprends pour l'essentiel des remarques de J.-G. Laplacherie, «Écriture et mise en page dans le Glossaire de Leiris ", dans Littérature, 51, octobre 1983, p. 28-40 et d'A.-M. Christin, L'image écrite [1995], Paris, Flammarion, 2009, p. 261-276.

18. Voir Langage tangage ou ce que les mots me disent, cit., p.111-114 et passim. Parmi les nombreuses contributions sur la relation entre signes verbaux et iconiques, je renvoie entre autres à M. Joly, Introduction à l'analyse de l'image [1993], Paris, Colin, 2009 ; A.-M. Christin, op. cit. ; L. Louvel - H. Scepi (dir.), Texte/image : nouveaux problèmes, Actes du colloque de Cerisy, P.U. de Rennes, 2005. ; et L. Sabourin (dir.), Poésie et illustration, P.U. de Nancy, 2008.

19. Cf. les deux articles au titre identique de «45, rue Blomet» : celui de l'artiste, de 1968, est recueilli dans A. Masson, Le rebelle du surréalisme. Écrits, F. Will-Levaillant (éd.), Paris, Hermann, 1976, p. 76-84 ; l'autre, de 1982, est repris dans M. Leiris, Zébrage, Paris, Gallimard, 1992, p. 219-229 (p. 227 pour la formule citée). Les nombreux textes de l'écrivain sur le peintre sont réunis dans M. Leiris, Écrits sur l'art, P. Vilar (éd.), Paris, Gallimard, 2011, p. 77-145. Leiris a même longuement glosé le nom de son ami dans un poème-hommage, publié en 1947 ; voir P. Vilar, "André Masson, la ligne et le nom: Narcisse et Écho", dans M. Leiris, Écrits sur l'art, cit., p. 152-164 (p. 156).

20. Cf. M. Leiris, Glossaire j'y serre mes gloses, éd. Yvert, cit. , p. 168-169 et L. Saphire-P. Cramer, André Masson, Catalogue raisonné des livres illustrés, Genève, P. Cramer, 1994, p. 46-47.

21. Les mots illustrés sont Assassinat, Caillou, Femme, Girasol, Héraclite, Lèvres, Lune, Méridienne, Métamorphose, Pensée, Ruine, Signe, Taureau, Veto et Waterloo. La seizième lithographie orne la couverture du recueil et se réfère au titre.

22. Voir F. Will-Levaillant, Catalogue des ouvrages illustrés par André Masson, 1924-1971, Paris, GiraudBadin, 1973, p. 23.

23. Dans la théorie classique de la traduction, ce schéma tripartite s'impose au moins à partir du XVII ${ }^{\mathrm{e}}$ siècle, avec Dryden et ensuite Goethe ; cf. G. Steiner, op. cit., p. 349-360.

24. "métamorphose - maladie métaphysique des morts. Formation métallique ? Mal morose ", M. Leiris, «En fête avec André Masson » [1977], Écrits sur l'art, cit., p. 135-140. Leiris aborde ce thème surréaliste, qui devient obsédant chez Masson (voir par exemple A. Masson, Le rebelle du surréalisme, cit., p. 222, n. 31).

25. Voir F. Vasarri, "Leiris alla ricerca del nome proprio", dans Rivista di letterature moderne e comparate, 2, 2007, p. 169-195; à ce propos, la glose de «flamme » en tant que "fluide mâle » est éloquente.

26. A. Masson, «Pensée » et « Signe » dans Minotaure, A. Breton (dir.), 11, printemps 1938, p. 53.

27. Pour une autre variante (une fleur sans bouche) voir aussi le dessin «La Pensée " (1938), reproduit dans le recueil collectif, André Masson [1940], Marseille, André Dimanche, 1993, s.i.p. [p. 39].

28. J. Derrida, « Des tours de Babel » [1985], dans Psyché. Inventions de l'autre, Paris, Galilée, 1987, p. 203-235 (en particulier p. 205-207).

29. A. Masson, « Mythologie de la nature » [1938] et « Anatomie de mon univers » [1940], dans Le rebelle du surréalisme, cit., p. 221-222. Françoise Will-Levaillant rattache en particulier le dernier syntagme aux «métaphores graphiques » des dessins pour Glossaire j'y serre mes gloses (Ibid., p. 221, note 24).

30. Cf. L. Saphire, André Masson. L'œuvre gravé, vol. I, New York, Blue Moon Press, p. 128, cité dans

L. Yvert, Bibliographie des écrits de Michel Leiris: 1924 à 1995, Paris, Jean-Michel Place, 1996, p. $100-101$. 
31. Je rappelle que René Magritte avait publié une sorte de manifeste sur cette question : "Les mots et les images ", dans La Révolution surréaliste, 12, 15 décembre 1929, p. 32-33.

32. «[...] le besoin de m'adonner, cygne et singe, à cette sorte de glossolalie, parler pour parler [... ] ", M. Leiris, Langage tangage ou ce que les mots me disent, cit., p. 96.

33. La définition est de Masson lui-même, vers 1973 ; cf. A. de Giry, «André Masson, peintrepoète », dans Mélusine, XII, 1991, p. 91-104.

\section{RÉSUMÉS}

Michel Leiris a défini ses jeux de mots en forme d'articles de dictionnaire comme des calembours poétiques. Glossaire j'y serre mes gloses est un recueil foncièrement intraduisible. Pourtant, un mouvement traductif le traverse dans plusieurs directions. D'abord, la glose n'est souvent que la traduction intralinguistique d'un mot isolé. En outre, Leiris s'aventure parfois dans Babel, en proposant des équivalents poétiques français de mots étrangers (traduction interlinguistique). Enfin, les illustrations d'André Masson pour l'édition de 1939 introduisent une troisième dimension, celle de la traduction intersémiotique. Masson assume jusqu'à un certain point la contrainte de transposer sur le plan iconique les jeux de la matière verbale. Son travail montre une variété de solutions, allant de la littéralité, axée sur le mot-entrée, à la fidélité au texte de la glose, que le dessin prolonge et développe, et jusqu'à la recréation libre et autonome, produisant de nouveaux jeux visuels. Il s'agit d'un cas marquant d'interaction texte-image, dans une mouvance intermédiaire entre le surréalisme et l'Oulipo.

\section{INDEX}

Mots-clés : traduction, jeu de mots, texte-image, poésie, avant-garde, Leiris (Michel), Masson (André), Glossaire j'y serre mes gloses 